\title{
Effects of endurance exercise and doxorubicin on skeletal muscle myogenic regulatory factor expression
}

Colin J. Quinn ${ }^{1,2}$

David S. Hydock 2,3

1 Aerospace and Operational Physiology, United State Air Force, Tyndall AFB, USA

2 School of Sport and Exercise Science, University of Northern Colorado, USA

3 Cancer Rehabilitation Institute, University of Northern Colorado, USA

Corresponding Author:

David S. Hydock

School of Sport and Exercise Science,

University of Northern Colorado

Gunter 2590, Box 39

501 20th Street

80639 Greeley, Colorado, USA

E-mail: david.hydock@unco.edu

\section{Summary}

Background: The skeletal muscle toxicity that accompanies the chemotherapy drug doxorubicin (DOX) may lead to cancer patient weakness and fatigue. This myotoxicity involves myogenic regulatory factor (MRF) disruption which alters muscle integrity and regeneration. Endurance exercise enhances MRF expression and thereby may mitigate DOX-induced MRF disruptions. This study examined the effects of endurance training and DOX treatment on myogenic regulatory factor (MRF) expression.

Methods: Male rats were exercise trained (EXER) or remained sedentary (SED) for two weeks. EXER and SED then received either DOX $(15 \mathrm{mg} / \mathrm{kg})$ or saline (SAL). Soleus, extensor digitorum longus (EDL), and diaphragm were excised 24 hours post injection, and MRF expression was analyzed.

Results: Significant Myf5 drug and activity effects were observed in the soleus with EXER+DOX expressing higher Myf5 than SED+DOX. A significant drug effect was detected in soleus MyoD, and a significant activity effect was detected in soleus Mrf4. No main effects or interactions were observed in the EDL, but in the diaphragm, a significant activity effect was observed for Myf5 with EXER+DOX expressing higher levels than SED+DOX.

Conclusion: Doxorubicin treatment increased soleus MRFs and exercise boosted MRF response in soleus and diaphragm suggesting that exercise may enhance regenerative signaling with DOX treatment.

Level of evidence: I b, individual randomized controlled trial.

KEY WORDS: anthracycline, diaphragm, fast muscle, slow muscle.

\section{Introduction}

Muscular weakness and fatigue with subsequent deterioration in activities of daily living are common side effects of chemotherapy treatments prescribed to cancer patients. The commonly used anthracycline antibiotic, doxorubicin (DOX; trade name: Adriamycin $\left.{ }^{\circ}\right)$, has been shown to have debilitating consequences typically associated with its cardiotoxicity. Free radicals formed by iron-catalyzed reactions are implicated in nuclear and mitochondrial damage which may induce cell death ${ }^{1}$. Much of the work surrounding DOX-induced injury focuses on the effects observed in the heart, but more recently, serious skeletal muscle damage resulting in decreased muscle size and function in response to DOX exposure has been reported ${ }^{2}$.

Skeletal muscle comprises a large part of the human body and is responsible for posture and locomotion as well as contributing to respiration. Skeletal muscle is unique in its plasticity as it has the capacity to alter its form following various stimuli. In adult skeletal muscle, myogenic regulatory factors (MRFs) guide satellite cells to restore muscle integrity in response to damage and stress. Activated satellite cells, expressing primary MRFs, form myoblasts, and differentiate into myotubes in response to secondary MRFs and reconstitute muscle fibers. The ability of skeletal muscle to repair itself and retain structure relies heavily on functional MRF proteins.

In vitro DOX exposure has been shown to decrease the ability of myoblasts to differentiate into myotubes $^{3}$. Additionally, MRF mRNA expression is compromised under the same conditions with an up-regulation of the MRF inhibitor, Id ${ }^{4}$. DOX has been shown to induce oxidative stress, leading to cellular damage and single-stranded DNA breaks. Beyond oxidative damage, genotoxic stress attributed to DOX leads to double-stranded DNA breaks. The primary MRF, My$\mathrm{OD}$, is fundamentally involved in myoblast DNA re- 
pair ${ }^{5}$, and its presence, along with the other MRFs, is critical for skeletal muscle regeneration following chemotherapy treatment including DOX. Although MRF mRNA following DOX exposure has been broadly investigated, protein expression has been examined less frequently.

Since DOX is effective as an anticancer drug, approaches to manage its toxicities have received attention, and one such intervention is exercise. The majority of this work, however, has centered on cardiotoxicity. We have shown previously that exercise training protects against DOX-induced cardiotoxicity ${ }^{6}$, but few reports exist exploring the effects of exercise on DOX-induced myotoxicity. It has been reported, however, that exercise preconditioning attenuates DOX-induced muscle dysfunction 7 , and the mechanisms of exercise protecting against DOX-myotoxicity have been attributed to oxidative stress, proteolysis, and autophagy signaling in skeletal muscle $e^{8,9}$ which are similar to mechanisms also explored with exercise and DOX cardiotoxicity, and thus mechanisms specific to skeletal muscle (such as those affecting MRF expression) may likely exist. Exercise has been shown to boost MRF signaling ${ }^{10}$ and thus may provide protection against DOX myotoxicity through enhanced MRF expression, but the effects of exercise prior to acute DOX exposure on skeletal muscle MRF expression have yet to be explored. Therefore, the purpose of this study was to examine the effects of endurance exercise training on the expression of the MRFs (MyoD, Myf5, myogenin, and Mrf4) following DOX administration. It was hypothesized that endurance exercise would upregulate MRF expression with DOX treatment thus providing a potential mechanism of protection against DOX myotoxicity.

\section{Methods and materials}

Ten-week-old male Sprague-Dawley rats (Envigo: Indianapolis, IN; $\mathrm{N}=47, \sim 300 \mathrm{~g}$ ) were housed in pairs under a 12:12 hour light-dark cycle at room temperature $\left(20 \pm 2{ }^{\circ} \mathrm{C}\right)$. Rats were provided food (Harlan Teklad 2026 rat chow) and distilled water ad libitum. All experimental procedures were approved by the Institutional Animal Care and Use Committee and were in compliance with the Animal Welfare Act guidelines. Additionally, this research project was conducted ethically according to international standards and as required by the journall ${ }^{11}$.

Rats were randomly assigned to the sedentary (SED) $(n=20)$ or exercise (EXER) $(n=27)$ group. The SED group was limited to normal cage activity for the duration of the study, and animals in the EXER group progressively trained on a motorized treadmill at 30 $\mathrm{m} / \mathrm{min}$ through week 1 and for one hour during week 2 (Tab. I). This two week treadmill protocol was used previously to protect against DOX-induced oxidative stress in skeletal muscle ${ }^{9}$. When necessary, rats were motivated by light electric shock at the rear of treadmill lanes. All EXER subjects completed the training protocol.

\section{Drug Treatment}

At the completion of the two week activity period, both SED and EXER animals remained sedentary for 24 hours prior to injections to remove the effects of acute exercise. After the 24 hour sedentary period, animals were subdivided to receive DOX or saline (SAL) injections: SED-SAL $(n=10)$, SED-DOX $(n=10)$, EXER-SAL $(n=13)$, EXER-DOX $(n=14)$. Subjects in the DOX group received a bolus i.p. $15 \mathrm{mg} / \mathrm{kg}$ injection of DOX hydrochloride (Bedford Labs: Bedford, $\mathrm{OH})$. Animals in the SAL group received an equivalent volume of $0.9 \%$ saline. Twenty four hours following injections, animals were anesthetized with sodium pentobarbital $(50 \mathrm{mg} / \mathrm{kg}$ ), and once a tail pinch reflex was absent, soleus (SOL), extensor digitorum longus (EDL), and a portion of the diaphragm (DIA) muscles were excised, flash frozen in liquid nitrogen, and stored at $-80^{\circ} \mathrm{C}$ for later biochemical analysis.

\section{Biochemical Analysis}

Frozen left hindlimb muscles (SOL and EDL) and DIA were homogenized and sonicated in radioimmunoprecipitation assay (RIPA) buffer (10:1) and protease enyme inhibitors (SigmaAldrich: St. Louis, MO). Homogenates were then centrifuged for 10 minutes at $3000 \mathrm{~g}$ at $4^{\circ} \mathrm{C}$. Total protein was quantified using the bicinchronic acid (BCA) assay and normalized with RIPA buffer.

Western blot analysis was conducted on muscle samples for the presence of MRFs in the SOL, EDL, and DIA. An equal volume of Lammeli buffer was added to samples. Samples were heated in boiling water for 2 minutes, then chilled on ice for 5 minutes before 46 $\mu \mathrm{g}$ of protein was separated using SDS-PAGE. Proteins were loaded onto $4-20 \%$ gradient Tris-Glycine gels (LifeTechnologies: Carlsbad, CA) and run at 125 constant voltage and $4 \mathrm{~mA}$ current for 2 hours in a Xcell II blot module (Invitrogen, LifeTechnologies). Proteins were transferred to 0.45 micron polyvinyli-

Table I. Treadmill exercise training protocol.

\begin{tabular}{llllllll}
\hline Day & $\mathbf{1}$ & $\mathbf{2}$ & $\mathbf{3}$ & $\mathbf{4}$ & $\mathbf{5}$ & $\mathbf{6}-\mathbf{7}$ & $\mathbf{8}-\mathbf{1 2}$ \\
\hline Speed (m/min) & 30 & 30 & 30 & 30 & 30 & (Rest) & 30 \\
Duration (min) & 10 & 20 & 30 & 40 & 50 & (Rest) & 60 \\
Incline (\%) & 0 & 0 & 0 & 0 & 0 & (Rest) & 0 \\
\hline
\end{tabular}


dene fluoride (PVDF) membranes over 90 minutes at 25 volts and $100 \mathrm{~mA}$. Protein transfer to PVDF membranes were ensured by the presence of a SeeBlue ${ }^{\circledR}$ Plus2 protein ladder (Novex, LifeTechnologies). Membranes were blocked for 1 hour in $15 \mathrm{~mL}$ of TBST $+5 \%$ milk and incubated with gentle agitation overnight in $10 \mathrm{~mL}$ of primary antibodies. Membranes were then washed in TBST three times for 5 minutes followed by incubation in appropriate species-specific secondary antibodies for 1 hour. After three more 5minute washes in TBST, membranes were prepared for protein band detection.

Detection was executed by enhanced chemiluminescence (ECL) (C-Digit, Li-Cor: Lincoln, NE), and ImageJ software (NIH: Bethesda, MD) was used to quantify the protein bands. Immediately before chemiluminescent imaging, $1.5 \mathrm{~mL}$ of luminol and enhancer (SuperSignal West Femto Maximum Sensitivity Chemiluminescent Substrate, ThermoScientific: Waltham, MA) was added to the membrane. The primary antibodies of interest included rabbit monoclonal MyoD, myogenin (Santa Cruz Biotechnology: Dallas, TX), Myf5, and Mrf4 (Abcam: Cambridge, MA). The rabbit monoclonal anti-GAPDH (Abcam) was used as a loading control. Molecular weights of protein bands were ensured in reference to a MagicMark ${ }^{\mathrm{TM}}$ XP standard ladder (Novex, LifeTechnologies). Secondary antibodies (Santa Cruz Biotechnology) corresponded to associated species (rabbit) and included horseradish peroxidase (HRP) for adequate reactivity.

\section{Statistical Analysis}

Data are presented as mean \pm SEM. A two-factor (Exercise X Drug) analysis of variance (ANOVA) was used to determine main effects and interactions for body mass, muscle mass, Myf5, MyoD, myogenin, and Mrf4 expression. If a significant F-value was observed, a Tukey's post-hoc testing was used to identify where differences existed. For all procedures, significance was set at $\mathrm{a}=0.05$.

\section{Results}

\section{General Observations}

Before beginning treadmill and sedentary conditions there were no differences between group in body mass $(p>0.05)$. Table II presents animal characteristics at the time of injection and at the time of sacrifice. At the time of injection (following two weeks of treadmill or sedentary conditions) a significant activity effect was observed $(p<0.05)$ with treadmill trained groups having lower body masses than sedentary animals. Post hoc testing revealed that body mass was higher in SED+SAL and SED+DOX than EXER+SAL $(p<0.05)$. At the time of sacrifice, a similar activity effect existed with lower body mass observed in EXER animals $(p<0.05)$. Post hoc testing revealed that EX$E R+S A L$ and EXER+DOX body masses were significantly lower than SED+SAL $(p<0.05)$.

There was no significant between group difference observed in SOL mass ( $p>0.05)$. Similarly, when SOL mass was expressed relative to body mass (muscle mass in $\mathrm{mg} \div$ body mass in grams), no significant difference was observed ( $p>0.05$, Tab. II). No activity effect, drug effect, or interaction was observed in absolute EDL mass ( $p>0.05)$, but when corrected for body mass (i.e., relative EDL mass), a significant activity effect was observed $(p<0.05)$. Although post hoc testing did not reveal precisely where differences existed, a trend toward EXER+DOX having a higher relative EDL mass than SED+DOX can be observed (Tab. II).

\section{Myogenic Regulatory Factor Expression}

Expression of Myf5, MyoD, Mrf4, and myogenin were analyzed in SOL, EDL, and DIA homogenates to assess the effects of a two-week endurance exercise protocol and acute DOX treatment. In the SOL, a significant activity $(p<0.05)$ and drug effect $(p<0.05)$ was observed for Myf5 suggesting that both endurance exercise and in vivo DOX treatment promote an up-

Table II. Animal characteristics.

\begin{tabular}{|c|c|c|c|c|}
\hline & SED+SAL & SED+DOX & EXER+SAL & EXER+DOX \\
\hline Injection Mass $(\mathrm{g})^{*}$ & $360 \pm 11$ a & $364 \pm 10^{a}$ & $322 \pm 5$ & $335 \pm 7$ \\
\hline Sacrifice Mass $(g)^{*}$ & $360 \pm 12^{a, b}$ & $354 \pm 9$ & $326 \pm 6$ & $329 \pm 6$ \\
\hline SOL Mass (mg) & $135 \pm 7$ & $140 \pm 4$ & $132 \pm 5$ & $133 \pm 5$ \\
\hline EDL Mass (mg) & $142 \pm 5$ & $138 \pm 8$ & $135 \pm 4$ & $141 \pm 4$ \\
\hline Relative SOL Mass (mg/g) & $0.374 \pm 0.012$ & $0.397 \pm 0.010$ & $0.403 \pm 0.011$ & $0.406 \pm 0.014$ \\
\hline Relative EDL Mass $(\mathrm{mg} / \mathrm{g})^{*}$ & $0.395 \pm 0.011$ & $0.389 \pm 0.017$ & $0.416 \pm 0.010$ & $0.432 \pm 0.016$ \\
\hline
\end{tabular}

SED+SAL, sedentary saline; SED+DOX, sedentary doxorubicin; EXER+SAL, exercise saline; EXER+DOX, exercise doxorubicin. Values are means \pm SEM.

${ }^{*}=$ significant activity effect $(p<0.05)$

$\mathrm{a}=$ significantly greater than EXER+SAL $(p<0.05)$

$\mathrm{b}=$ significantly greater than EXER+DOX $(p<0.05)$ 


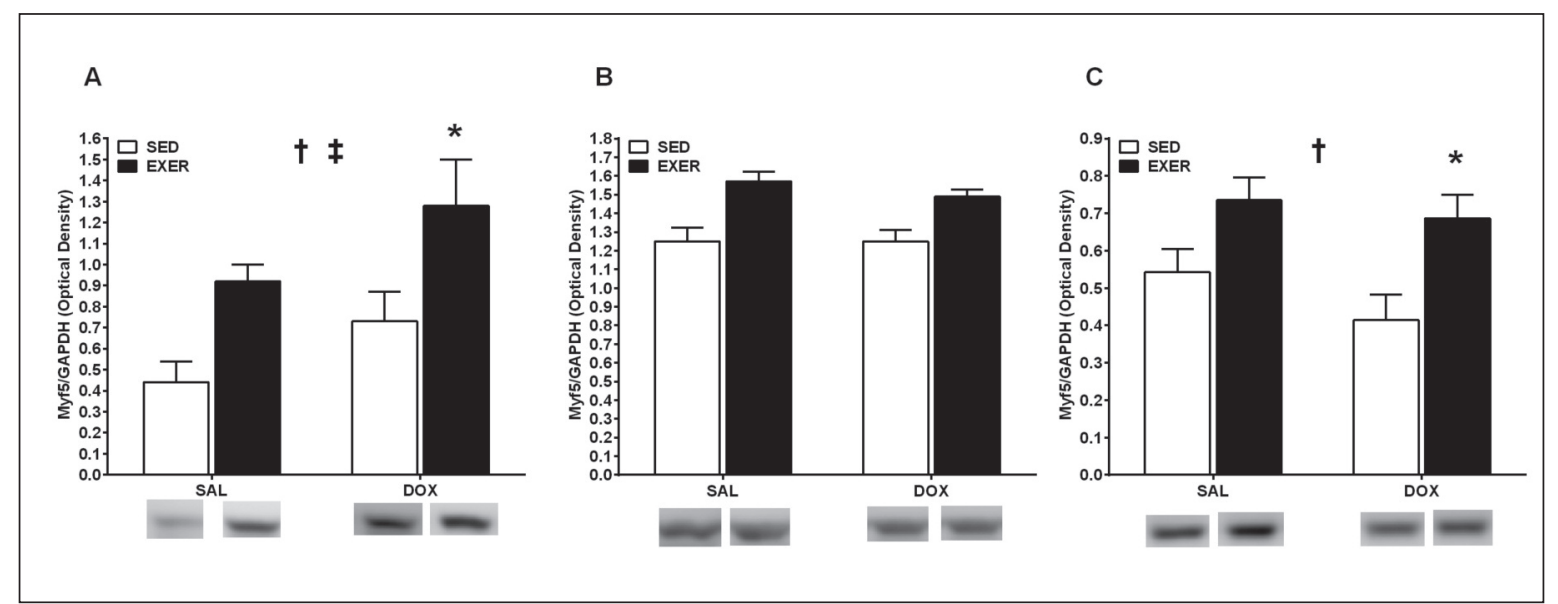

Figure 1. Myf5 expression in the soleus (A), extensor digitorum longus (B), and diaphragm (C). Data are mean \pm SEM. SED, sedentary; EXER, treadmill exercise trained; SAL, saline injection; DOX; doxorubicin injection.

$\dagger$ Main exercise effect $(p<0.05)$

$\ddagger$ Main drug effect $(p<0.05)$

* Significantly greater than SED+DOX

regulation of this primary MRF (Fig. $1 \mathrm{~A})$. Post hoc testing revelaed that EXER+DOX expressed significantly higher Myf5 than SED+DOX $(p<0.05)$. No significant main effects or interactions were observed with Myf5 expression in the EDL ( $>>0.05$, Fig.1 B), but in the DIA, a significant exercise effect was observed $(p<0.05)$ with EXER+DOX expressing significantly higher Myf5 than SED+DOX ( $p<0.05$, Fig. 1 C). In the SOL, a significant drug effect was observed with MyoD expression ( $p<0.05$, Fig. 2 A) which suggests that acute DOX exposure in vivo promotes upregulation of this primary MRF. Although post hoc testing did not reveal significant differences, a trend toward EXER+DOX having higher MyoD than SED+DOX can be observed. In the EDL and DIA, no significant activity effects, drug effects, or interactions were observed with MyoD expression ( $p>0.05$, Fig. 2 $B, C$ respectively). A significant exercise effect was observed with Mrf4 expression in the SOL $(p<0.05$, Fig. 3 A) suggesting that exercise upregulates expression of this secondary MRF; however, post hoc testing did not reveal individual group differences. No main effects or interactions were observed for EDL or DIA Mrf4 expression. In the SOL, no myogenin exercise effects, drug effects, or interactions were observed, but a trend toward SOL from exercised animals expressing higher myogenin than SOL from sedentary animals can be seen (Fig. 4 A). No main effects or interactions were observed for EDL or DIA myogenin expression (Fig. 4 B, C respectively).



Figure 2. MyoD expression in the soleus (A), extensor digitorum longus (B), and diaphragm (C). Data are mean \pm SEM. SED, sedentary; EXER, treadmill exercise trained; SAL, saline injection; DOX; doxorubicin injection. $\ddagger$ Main drug effect $(p<0.05)$ 




Figure 3. Mrf4 expression in the soleus (A), extensor digitorum longus (B), and diaphragm (C). Data are mean \pm SEM. SED, sedentary; EXER, treadmill exercise trained; SAL, saline injection; DOX; doxorubicin injection.

$\dagger$ Main exercise effect $(p<0.05)$

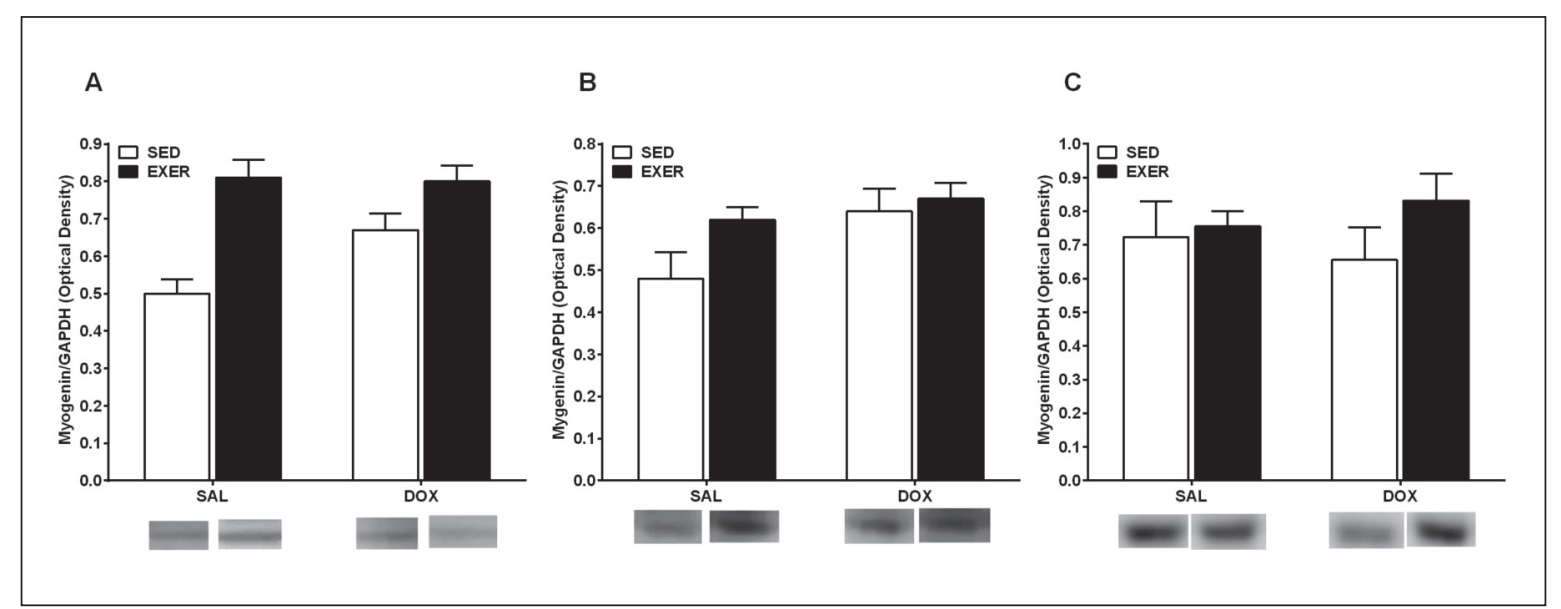

Figure 4. Myogenin expression in the soleus (A), extensor digitorum longus (B), and diaphragm (C). Data are mean \pm SEM. SED, sedentary; EXER, treadmill exercise trained; SAL, saline injection; DOX; doxorubicin injection. No significant main effects or differences $(p>0.05)$.

\section{Discussion}

To the Authors' knowledge, the current study is the first to explore the effects of short-term exercise training (two weeks) and short term in vivo DOX exposure (24 hours) on MRF expression in the type I (or slow) SOL, the type II (or fast) EDL, and DIA (mixture of slow and fast muscle). One of the novel findings of this study is that significant drug effects were observed with the primary MRFs (Myf5 and MyoD) in the SOL, and this upregulation is in contrast to what has been reported previously where in vitro DOX treatment downregulated MyoD expression and function thereby impairing the ability of embryonic cells (myoblasts and fibroblasts) to differentiate ${ }^{4}$. The time point for analysis in the current study was 24 hours following a bolus DOX injection which provides only a "snapshot" of the myogenic signaling response. It is likely that analysis of MRFs at a later time point (i.e., 5 days post DOX injection) would result in a response similar to that observed in the aforementioned in vitro study, and it is recommended that these later time points be explored in the future. Furthermore, DOX has been shown to inhibit MyoD binding to DNA in vitro thus preventing downstream differentiation ${ }^{4}$. These in vitro studies are helpful in understanding the impact that DOX has on the ability of immature muscle cells to develop through MRF signaling, but the effects of DOX on immature muscle cells in vitro do not translate to understanding the impact that DOX has on adult skeletal muscle. Furthermore, exploring the in vivo effects of DOX as with the current study allows for analysis of different skeletal muscle types as it has been shown previously that DOX has a differential effect on skeletal muscle types ${ }^{12}$. Nonetheless, DOX treatment impairs skeletal muscle function 
in vivo ${ }^{13}$, and cancer patients receiving DOX experience debilitating fatigue ${ }^{14}$. Because this debilitating fatigue can compromise a cancer patient's quality of life, there is need to obtain a better understanding of how DOX affects skeletal muscle.

Understanding how DOX impacts skeletal muscle MRF expression in vivo is an important step in better understanding DOX myotoxicity as a whole, but it first should be mentioned that the nature of DOX myotoxicity is more complex than merely accumulating in the myocyte leading to maladaptation. DOX does indeed have a "direct effect" on skeletal muscle through DOX accumulating in skeletal muscle and thereby directly interacting with skeletal muscle ${ }^{15}$, but in vivo DOX treatment may also have an "indirect effect" on skeletal muscle due its cardiotoxicity. DOX cardiotoxicity leads to reduced left ventricular function ${ }^{16}$ which can eventuate to heart failure ${ }^{17}$, and heart failure negatively impacts skeletal muscle ${ }^{18}$. The decreased blood flow to skeletal muscle promotes skeletal muscle wasting and weakness, and heart failure has been shown to disrupt skeletal muscle MRF expression ${ }^{19}$. Couple DOX's effects on the heart having an impact on skeletal muscle (indirect effect) with the direct effects of DOX on skeletal muscle, and the DOX effects on the primary MRFs observed in the current study may be due to a combination on DOX's direct and indirect effects on skeletal muscle.

Doxorubicin toxicity was once believed to be highly specific to the heart, and thus the majority of research exploring DOX toxicity and side effects have focused on the heart. It has now been commonly recognized, however, that DOX myotoxicity is a clinically relevant side effect. In vitro DOX treatment increases calcium release from isolated skeletal muscle sarcoplasmic reticulum ${ }^{20}$, and at the whole muscle level, in vitro DOX incubation interferes with actin-myosin interaction and decreases calcium sensitivity ${ }^{21}$. Furthermore, myotubes incubated with DOX have impaired maximal force production and maximal relaxation velocity as well as increased fatigue rates ${ }^{22}$. Our laboratory and others have reported that DOX accumulates in skeletal muscle in vivo ${ }^{15,23}$ which results in an excessive reactive oxygen species production. This oxidative stress prompts peroxidation, protein carbonyl formation, proteolysis ${ }^{9}$, apoptosis upregulation ${ }^{24}$, enhanced autophagy signaling ${ }^{8}$, and nuclear DNA damage ${ }^{25}$. Of utmost consequence to cancer patients receiving DOX, skeletal muscle damage at the cellular level leads to decreased force production and increased fatigue ${ }^{13,15}$, and cancer patients receiving DOX report severe to debilitating fatigue ${ }^{26}$.

Skeletal muscle is unique in its ability to adapt to stress and stimuli (myoplasticity) which is partially dependent on its capacity to signal the repair and restoration of the disrupted or injured cell. The process of myoplasticity involves a group of transcription factors called myogenic regulatory factors (MRFs) which, among other things, signal the activation, direction, and proliferation of satellite cells to restore muscle integrity in response to damage or trau$\mathrm{ma}^{27}$. This myoplasticity involves satellite cells which lie quiescently in the basal lamina under normal conditions; however, when a muscle fiber becomes damaged or stressed, satellite cells are activated, proliferate, and migrate to the site of injury where they fuse and incorporate themselves into the injury site to help repair the area. Satellite cell activation, proliferation, migration, fusion, and incorporation are directed by signaling from a group of basic helix-loop-helix transcription factors known as myogenic regulatory factors (MRFs). The MRF family includes the primary MRFs (Myf5 and MyoD) and the secondary MRFs (myogenin and Mrf4). In response to stress or injury, activated satellite cells initially express Myf5 which stimulates their proliferation ${ }^{28}$. Following proliferation, activated satellite cells express MyoD ${ }^{29}$ which is imperative in muscle regeneration ${ }^{30}$. Once the signaling from the primary MRFs (Myf5 and MyoD) has taken place, the secondary MRFs (myogenin and Mrf4) act to complete the process of muscle regeneration. Myogenin expression is essential in signaling the differentiation of satellite cells into myoblasts and fusion into myotubes ${ }^{31}$, and Mrf4 is then involved in signaling the maturation of the myotubes ${ }^{32}$.

Following 24 hours of treatment, DOX promoted an upregulation of Myf5 and MyoD in the SOL which suggests that this myotoxic insult of DOX stimulated signaling for repair in this type I muscle. No DOX effects were observed in the EDL or DIA which is consistent with the overall idea that DOX differentially affects skeletal muscle based on type. Although a trend toward a DOX effect with myogenin can be seen, no significant DOX effect was observed in the secondary MRFs, and this lack of an observed drug effect could be due to the early observation point in the current study. It is likely that DOX-induced alteration in myogenin or Mrf4 could be observed at later time points since these two transcription factors become active following upregulation and activation of the primary MRFs (Myf5 and MyoD).

There were also exercise effects observed for some MRFs analyzed with SOL from exercised rats having higher levels of Myf5 and Mrf4 than SOL from sedentary rats. Additionally, DIA from exercised rats expressed more Myf5 than DIA from sedentary rats. Beyond the significant main, exercise effects observed, it is important to mention that SOL and DIA Myf5 expression was significantly higher in EXER-DOX than SED-DOX suggesting that endurance exercise enhances SOL and DIA Myf5 expression in response to DOX. This "boost" in these MRFs may be one mechanism behind the exercise-induced protection against muscle dysfunction following DOX treatment ${ }^{7}$ by increasing the muscle's potential to repair damage promoted by DOX. It is also important to mention that DOX treatment can lead to diaphragm weakness and dysfunction ${ }^{33}$ which is of clinical concern as cancer patients receiving chemotherapy often experience respiratory muscle weakness and dyspnea ${ }^{34}$. The current study found that exercise prior to intraperitoneal DOX treatment enhanced DIA Myf5 expression suggesting that exercise may heighten DNA repair signaling with DOX potentially providing protection 
against respiratory distress. It should be noted, however, that the age of rats in the current study should be considered quite young. Although, the 10-week-old male rats used are sexually mature, the myogenic response to DOX may be different than that observed in older animals. It was reported previously that 6 month old rats had a somewhat different muscle repair signaling response to exercise and DOX at the mRNA level ${ }^{35}$ and muscle from 4-5-month-old rats, had a blunted inflammatory response with DOX and exercise ${ }^{36}$.

Nonetheless, the clinical application to the current study's finding should not be overlooked. Since a short-term exercise regimen (two weeks) prior to DOX administration enhanced MRF signaling in response to DOX, it is possible that exercise prescribed to patients prior to DOX treatment may be useful in minimizing myotoxicity through repair signaling mechanisms. Longer term exercise (10 weeks) prior to DOX treatment has been shown to attenuate DOX-induced skeletal muscle dysfunction ${ }^{7}$, but this clinical application is limited as cancer patients receiving DOX may not have 10 weeks to exercise train prior to treatment or be exercise trained prior to cancer diagnosis and/or treatment. Exploring the effects of short-term exercise prior to DOX treatment, as performed in the current study, is of importance as it is hoped that prescribing exercise prior to DOX treatment will become common practice. Short- term exercise prior to DOX treatment has been shown previously to attenuate cardiac dysfunction ${ }^{37}$, and although this type of exercise approach has been shown to mitigate markers of DOX myotoxicity ${ }^{35}$, more work should be done exploring how short-term exercise affects DOX-induced skeletal muscle dysfunction (i.e., reduced force production, increased fatigue rate). It is hoped that a deeper understanding of these effects will contribute to the understanding of how exercise can be best used to manage cancer treatment side effects in patients.

In closing, the current study explored the effects of short-term exercise training (two weeks) and acute in vivo DOX exposure (24 hours) on the expression of primary and secondary MRFs. DOX promoted an upregulation in the primary MRFs Myf5 and MyoD in the SOL suggesting early repair signaling in slow, or type I, muscle. The observed exercise effects, however, suggest that endurance training enhances Myf5 (SOL and DIA) and Mrf4 (secondary MRF in the SOL) thereby potentially contributing to augmented repair of DOX-induced damage. It is recommended that later time points following DOX administration be examined in the future as it is likely that longer exposure to DOX will lead to MRF downregulation and/or a different primary and secondary MRF expression profile. It is also recommended that future studies explore the expression of negative regulators of skeletal muscle repair and growth such as inhibitors of DNA binding (Id), myostatin (GDF-8), and muscle ring finger-1 (Murf-1) to gain a more complete understanding of the signaling and regulation of skeletal muscle repair. Although Kavazis et al. ${ }^{35}$ reported that exercise atten- uated DOX-induced Murf-1 mRNA downregulation, there is a need to assess Murf- 1 and similar negative regulators at the protein, or transcription factor, level which directly impacts the regulation of the myogenic response. As such, further exploration of these negative regulators would help discern the impact of exercise in modulating DOX-induced skeletal muscle damage.

\section{References}

1. DeAtley SM, Aksenov MY, Aksenova MV, Harris B, Hadley R, Cole Harper $P$, et al. Antioxidants protect against reactive oxygen species associated with adriamycin-treated cardiomyocytes. Cancer Lett. 1999;136:41-46.

2. Gilliam LA, Fisher-Wellman KH, Lin CT, Maples JM, Cathey $\mathrm{BL}$, Neufer PD. The anticancer agent doxorubicin disrupts mitochondrial energy metabolism and redox balance in skeletal muscle. Free radical biology \& medicine. 2013;65:988-996.

3. Kurabayashi M, Jeyaseelan R, Kedes L. Antineoplastic agent doxorubicin inhibits myogenic differentiation of $\mathrm{c} 2$ myoblasts. The Journal of biological chemistry. 1993;268:5524-5529.

4. Kurabayashi M, Jeyaseelan R, Kedes L. Doxorubicin represses the function of the myogenic helix-loop-helix transcription factor myod. Involvement of id gene induction. The Journal of biological chemistry. 1994;269:6031-6039.

5. Kobayashi J, Antoccia A, Tauchi H, Matsuura S, Komatsu K. Nbs1 and its functional role in the DNA damage response. DNA Repair (Amst). 2004;3:855-861.

6. Hydock DS, Lien CY, Jensen BT, Schneider CM, Hayward R. Exercise preconditioning provides long-term protection against early chronic doxorubicin cardiotoxicity. Integr Cancer Ther. 2011;10:47-57.

7. Bredahl EC, Pfannenstiel KB, Quinn CJ, Hayward R, Hydock DS. Effects of exercise on doxorubicin-induced skeletal muscle dysfunction. Med Sci Sports Exerc. 2016.

8. Smuder AJ, Kavazis AN, Min K, Powers SK. Exercise protects against doxorubicin-induced markers of autophagy signaling in skeletal muscle. J Appl Physiol (1985). 2011;111:11901198.

9. Smuder AJ, Kavazis AN, Min K, Powers SK. Exercise protects against doxorubicin-induced oxidative stress and proteolysis in skeletal muscle. J Appl Physiol (1985). 2011;110:935-942.

10. Aguiar AF, Vechetti-Junior IJ, Alves de Souza RW, Castan EP, Milanezi-Aguiar RC, Padovani CR, et al. Myogenin, myod and igf-i regulate muscle mass but not fiber-type conversion during resistance training in rats. Int J Sports Med. 2013;34:293-301.

11. Padulo J, Oliva F, Frizziero A, Maffulli N. Muscles, Ligaments and Tendons Journal - Basic principles and recommendations in clinical and field science research: 2016 update. MLTJ. 2016;6(1):1-5.

12. Ertunc M, Sara $Y$, Korkusuz P, Onur R. Differential contractile impairment of fast- and slow-twitch skeletal muscles in a rat model of doxorubicin-induced congestive heart failure. Pharmacology. 2009;84:240-248.

13. Hydock DS, Lien CY, Jensen BT, Schneider CM, Hayward R. Characterization of the effect of in vivo doxorubicin treatment on skeletal muscle function in the rat. Anticancer research. 2011;31:2023-2028.

14. Liu J, Tu D, Dancey J, Reyno L, Pritchard KI, Pater J, et al. Quality of life analyses in a clinical trial of dppe (tesmilifene) plus doxorubicin versus doxorubicin in patients with advanced or metastatic breast cancer: Ncic ctg trial ma.19. Breast Cancer Res Treat. 2006;100:263-271.

15. Hayward R, Hydock D, Gibson N, Greufe S, Bredahl E, Parry $\mathrm{T}$. Tissue retention of doxorubicin and its effects on cardiac, 
smooth, and skeletal muscle function. Journal of physiology and biochemistry. 2013;69:177-187.

16. Hayward R, Hydock DS. Doxorubicin cardiotoxicity in the rat: An in vivo characterization. Journal of the American Association for Laboratory Animal Science: JAALAS. 2007;46:20-32.

17. Belham M, Kruger A, Mepham S, Faganello G, Pritchard C. Monitoring left ventricular function in adults receiving anthracycline-containing chemotherapy. European journal of heart failure. 2007;9:409-414.

18. Dalla Libera L, Vescovo G, Volterrani M. Physiological basis for contractile dysfunction in heart failure. Current pharmaceutical design. 2008;14:2572-2581.

19. Martinez PF, Okoshi K, Zornoff LA, Carvalho RF, Oliveira Junior SA, Lima AR, et al. Chronic heart failure-induced skeleta muscle atrophy, necrosis, and changes in myogenic regulatory factors. Medical science monitor: international medical journal of experimental and clinical research. 2010;16:BR374383.

20. Abramson JJ, Buck E, Salama G, Casida JE, Pessah IN. Mechanism of anthraquinone-induced calcium release from skeletal muscle sarcoplasmic reticulum. The Journal of biological chemistry. 1988;263:18750-18758.

21. De Beer EL, Finkle H, Voest EE, Van Heijst BG, Schiereck P. Doxorubicin interacts directly with skinned single skeletal muscle fibres. European journal of pharmacology. 1992;214:97100.

22. van Norren K, van Helvoort A, Argiles JM, van Tuijl S, Arts K, Gorselink M, et al. Direct effects of doxorubicin on skeletal muscle contribute to fatigue. $\mathrm{Br} J$ Cancer. 2009;100:311-314.

23. Fabris S, MacLean DA. Skeletal muscle an active compartment in the sequestering and metabolism of doxorubicin chemotherapy. PloS one. 2015;10:e0139070.

24. Yu AP, Pei XM, Sin TK, Yip SP, Yung BY, Chan LW, et al. Acylated and unacylated ghrelin inhibit doxorubicin-induced apoptosis in skeletal muscle. Acta physiologica. 2014;211:201-213.

25. Puri PL, Medaglia S, Cimino L, Maselli C, Germani A, De Marzio E, et al. Uncoupling of p21 induction and myod activation results in the failure of irreversible cell cycle arrest in doxorubicin-treated myocytes. Journal of cellular biochemistry. 1997;66:27-36.

26. Gilliam LA, St Clair DK. Chemotherapy-induced weakness and fatigue in skeletal muscle: The role of oxidative stress. Antioxidants \& redox signaling. 2011;15:2543-2563.
27. Brown LA, Lee DE, Patton JF, Perry RA, Jr., Brown JL, Baum $\mathrm{Jl}$, et al. Diet-induced obesity alters anabolic signalling in mice at the onset of skeletal muscle regeneration. Acta physiologica. 2015;215:46-57.

28. Crist CG, Montarras D, Buckingham M. Muscle satellite cells are primed for myogenesis but maintain quiescence with sequestration of myf5 mrna targeted by microrna-31 in mrnp granules. Cell stem cell. 2012;11:118-126.

29. Joanisse S, McKay BR, Nederveen JP, Scribbans TD, Gurd BJ, Gillen JB, et al. Satellite cell activity, without expansion, after nonhypertrophic stimuli. American journal of physiology. Regulatory, integrative and comparative physiology. 2015; 309:R1101-1111.

30. Sabourin LA, Girgis-Gabardo A, Seale P, Asakura A, Rudnicki MA. Reduced differentiation potential of primary myod-/myogenic cells derived from adult skeletal muscle. The Journal of cell biology. 1999;144:631-643.

31. Onofre-Oliveira PC, Santos AL, Martins PM, Ayub-Guerrieri D, Vainzof M. Differential expression of genes involved in the degeneration and regeneration pathways in mouse models for muscular dystrophies. Neuromolecular medicine. 2012;14:7483.

32. Gayraud-Morel B, Chretien F, Flamant P, Gomes D, Zammit PS, Tajbakhsh S. A role for the myogenic determination gene myf5 in adult regenerative myogenesis. Developmental biology. 2007;312:13-28.

33. Gilliam LA, Moylan JS, Ann Callahan L, Sumandea MP, Reid MB. Doxorubicin causes diaphragm weakness in murine models of cancer chemotherapy. Muscle \& nerve. 2011; 43:94-102.

34. Webb K, Travers J, Amjadi K, McBride I, Dillon K, Laveneziana $\mathrm{P}$, et al. Mechanisms of exertional dyspnea in patients with cancer. Journal of Palliative Care. 2008:24:196-197.

35. Kavazis AN, Smuder AJ, Powers SK. Effects of short-term endurance exercise training on acute doxorubicin-induced foxo transcription in cardiac and skeletal muscle. J Appl Physiol (1985). 2014;117:223-230.

36. Huang SC, Wu JF, Saovieng S, Chien WH, Hsu MF, Li XF, et al. Doxorubicin inhibits muscle inflammation after eccentric exercise. J Cachexia Sarcopenia Muscle. 2017;8:277-284.

37. Wonders KY, Hydock DS, Schneider CM, Hayward R. Acute exercise protects against doxorubicin cardiotoxicity. Integr Cancer Ther. 2008;7:147-154. 